Meta

Journal des tradlucteurs

Translators' Journal

\title{
Conflits de juridiction
}

\section{La double signature du texte traduit}

\section{Sherry Simon}

Volume 34, numéro 2, juin 1989

URI : https://id.erudit.org/iderudit/004152ar

DOI : https://doi.org/10.7202/004152ar

Aller au sommaire du numéro

Éditeur(s)

Les Presses de l'Université de Montréal

ISSN

0026-0452 (imprimé)

1492-1421 (numérique)

Découvrir la revue

Citer cet article

Simon, S. (1989). Conflits de juridiction : la double signature du texte traduit. Meta, 34(2), 195-208. https://doi.org/10.7202/004152ar d'utilisation que vous pouvez consulter en ligne.

https://apropos.erudit.org/fr/usagers/politique-dutilisation/ 


\title{
CONFLITS DE JURIDICTION
}

\author{
La double signature du texte traduit ${ }^{\star}$
}

SHERRY SIMON

Université Concordia, Montréal, Canada

\author{
«His works will stand distinguished; his the sole Property of them; which Property alone \\ can confer the noble title of an Author.» \\ Edward Young, Conjectures on Original Composition \\ «Some, un Traducteurn'a james le nom d'Auteur.» \\ Jacques Pelletier, Art poétique.
}

Le nom du traducteur** est historiquement le lieu d'une insignifiance radicale. Rattaché au texte traduit d'une manière analogue mais non identique à celle qui lie le nom de l'auteur à son texte, le nom du traducteur n'a ni contenu ni fonction précis. Si depuis la fin de la Renaissance le nom de l'auteur remplit des fonctions devenues nécessaires à la mise en circulation et à la réception du livre (sa fonction classificatoire, sa fonction explicative, sa fonction légale), le nom du traducteur est un supplément dont la fonction semble largement honorifique. Les doutes sur la signification réelle du nom du traducteur se reflètent jusque dans l'imprécision quant à la place physique accordée à ce nom dans l'espace d'un livre et de l'imprimé en général.

Dans l'indétermination de ces fonctions, le nom du traducteur reflète le statut historiquement ambigu du traducteur comme sujet de l'écriture. Tributaire de la notion d'auteur, chronologiquement autant que logiquement, «le traducteur» constitue en effet une position dont le contenu varie d'après l'époque et le type de discours. En cela, l'histoire du nom du traducteur est symétrique à celle du nom de l'auteur, dont Michel Foucault dans son étude séminale nous a montré le caractère profondément historiquel. Le nom du traducteur devient donc un indice du lien entre la théorisation des traductions. La signification donnée au nom du traducteur (ou ce qu'on pourrait appeler la «fonctiontraducteur» par analogie avec la «fonction-auteur» étudiée par Foucault) révèle à la fois l'appartenance individuelle / culturelle du texte traduit à la manière dont cette appartenance est constituée dans l'économie générale des biens culturels.

Nous nous proposons dans cette étude de mettre en question l'insignifiance du nom du traducteur en essayant de lui donner une dimension historique et théorique. Dans un premier temps nous étudierons donc la généalogie de la fonction-traducteur, nous noterons quelques aspects importants de son émergence en tant que sujet de l'écriture à l'époque de la Renaissance. Dans un deuxième temps nous essaierons de comprendre la nature du lien (de propriété, de responsabilité juridique, mais aussi de responsabilité créatrice) qui unit aujourd'hui le nom du traducteur à sa production.

Ce travail se veut une contribution théorique à l'étude du rapport historique entre ces deux sujets de l'écriture que sont l'auteur et le traducteur. En effet, l'historicisation de l'activité de la traduction ne peut se faire sans que d'autres pratiques de l'écriture et leur conceptualisation ne soient mises en parallèle, sans que l'interdépendance de la fonctiontraducteur et de la fonction-auteur ne soient mises en évidence. 
Comment traiter un acte discursif caractérisé par la solidarité d'une structure mentale et d'un fait de langage, peut-être universels, et d'une pratique institutionnelle, assurément conditionnelle dans ses modalités diverses?

Antoine Compagnon, la Seconde main.

\section{GÉNÉALOGIE : L'émergence du sujet-traducteur}

La traduction en tant que processus d'échange linguistique existe depuis les premières civilisations de l'écrit. L'activité de traduction n'a pas toujours, cependant, été conçue comme une activité translinguistique unique. Il semble en effet que ce sont les humanistes italiens au début de la Renaissance, qui ont introduit le vocabulaire et la conception unitaire de la traduction qui prévaut actuellement dans les langues européennes ${ }^{2}$. La terminologie des Grecs, des Romains et surtout du Moyen Âge chrétien suggère que les conceptions pré-humanistes de la traduction rejoignent la théorisation contemporaine en décrivant la traduction moins comme une opération translinguistique que comme une opération transtextuelle.

Les termes qui désignent l'activité de traduction en grec et en latin indiquent que la traduction était conçue, d'une part, comme un cas particulier d'une activité plus générale (l'exégèse) et, d'autre part, comme une activité elle-même multiple. Le grec herméneuein veut dire à la fois «expliquer» et «traduire»; le latin «interpres» désigne le traducteur et l'exégète (Pépin: 295; Cave: 35). En effet, le concept culturel de la traduction est presque inexistant chez les Grecs jusqu'à l'époque alexandrine (Folena: 62). Les Romains par contre possèdent un nombre important d'expressions pour désigner surtout la traduction poétique et littéraire (verto, converto, transverto, imitari, explicare, exprimere, reddere et plus tard translatare). Le processus de récupération et d'assimilation de la culture grecque fera partie d'une réflexion théorique sur l'imitation (Cave: 57).

C'est le Moyen Age cependant qui nous fournit l'exemple le plus clair et le mieux documenté d'une conceptualisation pluridimensionnelle de la traduction. Face à une multiplicité de positions possibles dans la production du discours, la position du traducteur devient elle-même multiple. Là où «le travail d'écriture, celui du juriste, ou du médecin, mais aussi celui de l'historien, du philosophe moraliste, sans parler bien sûr du théologien, était discours sur du discours, agencement de textes préalables [...] sans que puisse se repérer clairement l'origine des différentes paroles, il n'y avait guère de raison pour que le traducteur-interprète-commentateur occupe une place distincte, ni que la traduction ait à être pensée comme un objet occupant une fonction spécifique dans le champ de la production écrite» (Guillerm: 13). Tout écrit destiné à introduire étant perfectible, «du moment qu'on le transcrit et qu'on le traduit, on ne voit aucune raison pour ne pas le modifier au goût du jour ou l'améliorer en le complétant de renseignements puisés à d'autres sources» (Monfrin : 217). La distinction entre la production et la transformation du discours s'effrite.

Le statut de la traduction est également rendu problématique par l'absence de frontières linguistiques stables. Peut-on dire que les Merveilles du monde de Marco Polo, écrits dans un curieux mélange de français et de franco-vénitien, ont été traduits en français, en vénitien et en toscan? Peut-on parler de traduction dans un monde intellectuel où le multilinguisme est chose courante et où l'écrivain s'exprime en langues diverses selon le sujet et le ton recherché? (Terracini : 57; Amos : 8 ; Leonard Forster : passim).

Le foisonnement terminologique mis en lumière par Folena confirme la multiplicité des concepts de la traduction face au type de textes et aux langues différentes, «selon que la langue de départ soit le latin ou un vulgaire, ou par rapport aux niveaux culturels et aux genres littéraires, textes religieux et didactiques, juridiques, historiques, poétiques» 
(Folena: 65). On distingue donc entre «volgarizzare et transporre» en italien, et en français, entre «espondre», «translater», «turner», «metre en romanz», «enromanchier». La plus nette ligne de partage concerne la distinction entre la traduction verticale (traductions des écrits saints et des auctores) et la traduction horizontale (traduction non pas à partir des «langues difficiles» mais entre ce que Don Quixote appelait les «langues faciles» (Terracini : 57).

Le «traducteur» est donc constitué d'une multiplicité de fonctions dont le caractère particulier est défini surtout par le type de texte en question. En cela il reflète la diversité de statuts dont jouissent les textes selon qu'ils sont le produit d'auctores ou qu'ils circulent de façon anonyme.

L'introduction du terme «traducere» par les humanistes italiens (et en particulier par Leonardi Bruni, De interpretatione recta, 1420) met fin à cette abondance terminologique et introduit pour la première fois une conception unitaire de la traduction (Folena : 102 ; Norton). La spécificité textuelle de la traduction sera oubliée, et la traduction désignera dorénavant toute activité translinguistique écrite.

\section{La hiérarchisation obligatoire}

Du passage de la traduction du Moyen Âge à la Renaissance, nous comprenons que la conception moderne de la traduction repose sur des conditions minimales qui constituent autant de frontières : 1) l'existence des langues bien délimitées (les grammairiens de la Renaissance désigneront cette délimitation par le concept de proprietas; 2) l'existence du texte clos; et 3) l'existence de l'auteur comme garant de l'authenticité du discours. Pour que le traducteur existe, il faut qu'il y ait langue, texte et auteur. Traducteur et auteur $^{3}$, dans la forme que nous leur connaissons, naissent en même temps et face aux mêmes réalités de la production du discours.

Cette co-naissance sera marquée, et dès le début, par une relation hiérarchique. Même si nous savons que l'on accorde une grande importance à la traduction à l'époque de la Renaissance, qu'elle est encouragée de manière officielle par l'État et le pouvoir royal en France et en Angleterre (Guillerm: 13; Derrida 1984: 97 ; Rosenberg: 154), que traducteurs et écrivains ont recours aux mêmes mécènes, vendent leurs livres aux même éditeurs, et produisent des biens considérés de la même manière comme des sources de richesses ${ }^{4}$ (Sheavyn; Bennett; Guillerm), le rapport entre l'écrivain et le traducteur sera théorisé comme un rapport hiérarchique. Les travaux de Luce Guillerm sur les préfaces françaises de 1530 à 1560 montrent que

le procès prétendu fait à la traduction est donc bien, et fort tôt, soustendu par la constitution d'une hiérarchie privilégiant l'œuvre originale, la mise en place d'une corrélation inventionpropriété-liberté (surprenante justement dans la mesure où l'idée nous est devenue trop familière) et enfin l'évacuation de l'idée de «travail», rejetée sur des opérations secondes, et secondaires, traduction, commentaires, interprétations (Guillerm: 12).

Ce qui émerge à la Renaissance c'est donc la définition de la position du traducteur en tant que sujet de l'écriture. Agent de la production du texte au même titre que l'auteur (et jouissant même à un certain moment d'un statut économique comparable à celui-ci), il se définit tout de même comme son négatif.

La reduction ad unum de la traduction (Folena, Norton), la diffusion sémantique dont souffrira le terme, aura donc plusieurs conséquences. La traduction deviendra un terme à la fois descriptif et normatif, sujet à des «pulsations sémantiques» (Moser: 7) dont les éléments sont rarement élucidés. Il n'existera jusqu'à nos jours que deux champs sémantiques où figure la traduction: d'une part, la triade traduction / version / paraphrase (surtout utilisées dans un contexte pédagogique et définie avec autorité dans l'article «tra- 
duction» de l'Encyclopédie de Diderot) et, d'autre part, la distinction entre la traduction littéraire et la traduction non littéraire - énoncée dès du Bellay et maintenue avec très peu de variations à travers l'époque romantique jusqu'à nous (Moser 1985).

En effet, en l'absence d'un vocabulaire qui pourrait rendre compte des divers lieux d'insertion de la subjectivité du traducteur, le discours sur la traduction depuis la Renaissance devient essentiellement un débat autour de la problématique de l'autorisation. L'enjeu essentiel du discours sur la traduction devient la délimitation du pouvoir respectif de l'auteur et du traducteur. La notion de fidélité «s'exprime, qu'on l'accepte ou la rejette, en termes d'acceptation ou de refus d'une relation d'autorité» (Guillerm: 16). Cette relation se reflète tout au long de la tradition des représentations métaphoriques privilégiées: le conflit de la traduction est dramatisé dans l'arène matérielle du territoire ( «But slaves we are, and labour on another man's plantation; we dress the vineyard, but the wine is the owner's5.» John Dryden preface to Aeneid [1697]), mais également dans des figures employant le registre sexuel («...since all translations are reputed femalls delivered at second hand». John Florio, preface to Montaigne's Essays.)

À défaut d'autres schèmes de référence, donc, le discours sur la traduction depuis la Renaissance adopte comme axe principal le rapport d'autorité entre le traducteur et l'auteur. C'est ce qui donne à ce discours son caractère curieusement a-historique, immobile, répétitif. Le sujet de la traduction qui sera articulé à la Renaissance aura-t-il en fait une histoire?

\section{LE POUVOIR DU TRADUCTEUR DANS L'HISTOIRE : l'exemple des Belles Infidèles}

La phrase que je dis ou que j'écris m'appartient, elle est à moi. C'est pourquoi il est nécessaire que la circulation de phrases sur le marché soit surveillée. (A. Compagnon, la Seconde main.)

Le traducteur, dès son émergence comme sujet d'écriture, dès sa définition comme agent d'un processus discursif unique, définira son travail en termes de son rapport hiérarchisé et conflictuel à l'auteur. Peut-on prendre ce discours au sérieux? Comment donc peut-on expliquer l'attitude tantôt humble, tantôt présomptueuse du traducteur?

On pourrait être tenté de dire que le discours d'autorisation limitée (la base de la très grande partie des commentaires de traducteurs sur leur propre travail, voir Horguelin 1981 et T. Steiner 1975 par exemple) est un discours rituel et proprement insignifiant. Largement confiné à des préfaces, il a un but souvent contradictoire puisque mettant en valeur le nom du traducteur mais insérant son travail dans un cadre de justifications étroites. Pour restituer ou créer la signification de ce discours il ne suffit pas alors d'étudier ces textes en isolation: il faut situer le rapport entre traducteur et auteur dans le contexte d'autres pratiques de l'écriture à l'époque. Alors peut apparaître non pas une histoire de la traduction, linéaire et lisse, mais bien un certain nombre de rapports possibles à l'écriture définie par des paramètres constants.

Louis Kelly, dans The True Interpreter, a déjà proposé une «histoire» de la traduction qui réponde à ces exigences. Il étudie le discours sur la traduction dans son rapport à deux grandes conceptions du langage: le langage-instrument et le langage-création. Bien que son analyse soit très riche, elle repose surtout sur la définition de la traduction comme une activité linguistique et non pas proprement textuelle. À partir de l'exemple du discours de l'école des «Belles infidèles» en France et en empruntant les concepts de «rhétorique» et de «commentaire» à Michel Charles, nous voudrions proposer un complément plus proprement textuel au travail de Louis Kelly. 
Le discours de la secte perrotine et plus tard des traducteurs «libres» en Angleterre fait irruption dans le courant tranquille des propos des traducteurs sur leur travail depuis la Renaissance; soudain le traducteur s'affirme, revendique son pouvoir, se dresse contre celui qu'il proclame quand même être son idole: l'auteur. Il n'y aurait pas que l'auteur qui fait montre de «style» et de «pensée». Ainsi d'Ablancourt (préface à Arrian): «cet Autheur est sujet à des repetitions frequentes et inutiles, que ma langue ny mon stile ne peuvent souffrir» et Jules Pilet de la Ménardière (Zuber: 83 ) : «J'ai pris la liberté de mesler souvent mes pensées parmy celles de notre Autheur».

Qu'est-ce qui explique l'insistance de la revendication des traducteurs perrotins et les débats importants autour de questions de traduction? Bien que la pratique de la traduction dans certains cas n'ait guère changé depuis le siècle précédent (de Blignières), l'inflation au niveau du discours sur la traduction témoigne d'une crise dans le rapport entre l'auteur et le traducteur. Cette expression de maîtrise de la part des traducteurs est d'autant plus difficile à comprendre qu'on assiste à cette même époque à des changements profonds dans les notions de propriété littéraire.

En effet, Antoine Compagnon a montré dans sa très importante étude sur l'histoire de la citation, la Seconde main, que la pratique de la citation depuis l'Antiquité a traversé trois «régimes» différents qui impliquent trois conceptions différentes de l'économie de l'écrit. Après l'Antiquité, le Moyen Âge et la période de transition que représente la Renaissance, c'est seulement à l'époque classique que s'instaure l'institution de la «périgraphie» et le texte «homéostatique».

La périgraphie fait du paysage textuel une culture bocagère; elle met un terme au débat, au délire quant à l'utilisation du déjà dit, elle résout une fois pour toutes les litiges usufruitiers car elle régularise, dans le double sens de mettre en règle et de rendre régulier, le fonctionnement de la machine à écrire ou à discourir. (Compagnon : 349)

C'est au long du $17^{\mathrm{e}}$ siècle que se fixeront les protocoles d'appartenance du livre (sans quoi le livre sera désormais disqualifié) et que s'élaboreront les justifications philosophiques (par Locke et Kant) du droit d'auteur. Le $17^{\mathrm{e}}$ siècle distinguera entre le livre comme objet et l'utilisation du texte lui-même; il fera de l'auteur celui qui contrôle la reproduction du texte. Le texte devient «propriété»: «chaque petit propriétaire du texte s'entoure d'un mur, d'une périgraphie. Le texte est enceint, l'auteur est maître de lui et de son territoire» (Compagnon : 356).

Qu'est-ce qui permet aux traducteurs perrotins de forcer le mur des textes anciens et de se proclamer maîtres du territoire? Pourquoi est-ce que le discours et la pratique d'Ablancourt sont acceptés à son époque et rigoureusement calomniés à d'autres? Roger Zuber dans son importante étude, les Belles infidèles et la formation du goût classique, montre qu'à l'époque classique il s'opère une étonnante coïncidence entre les critères de légitimisation de la «création» et de la «traduction». La notion de «rhétorique» telle qu'employée par Michel Charles (1985: 12) rend compte de cette coïncidence: la rhétorique, au contraire du commentaire, «conçoit le discours antérieur non pas comme un texte que l'on consigne et relit mais comme un instrument à fabriquer d'autres discours». Ainsi, création et traduction se comportent de la même manière vis-à-vis des auteurs anciens, encore références obligatoires: auteur et traducteur deviennent des «représentants modernes» de l'auteur ancien. Dans les deux cas la production est orientée vers le "goût» du public et le génie de la langue acquiert une valeur sacrée (Charles : 211).

L'époque est dominée par une esthétique de la production littéraire qui néglige les exigences des cultures du commentaire, de l'exégèse scholastique, de la sacralité textuelle. Imitation, traduction et littérature ne font qu'un. Cette constatation a été reprise, sur le mode d'une critique virulente, par de Blignières dans sa célèbre Éloge d'Amyot en 1851. 
Pour la «nouvelle scholastique» du $19^{e}$ siècle, cette cöncidence représentait un scandale. «Il semblait presque en effet qu'on accordait les mêmes droits sur le génie ancien à l'auteur qui s'en inspirait dans une création nouvelle, et à l'écrivain qui le représentait dans une traduction» (de Blignières : 249). Si de Blignières avoue que les manières de traduire d'Amyot et d'Ablancourt sont identiques, il n'approuve que la première. Sa critique de d'Ablancourt s'adresse à un discours et une pratique de la traduction qui sont historiquement inappropriés 6 . Le traducteur doit accepter «la sujétion de son rôle et mettre modestement tout son art à bien réfléchir celui de son auteur... Là se borne son office» (de Blignières : 263).

Le vocabulaire de l'autorisation répond à la question: comment peut-on produire un texte à partir d'un autre? Contrairement à ce qu'on aurait pu imaginer, le discours sur la traduction de façon générale ne rend pas compte explicitement des critère de légitimisation des stratégies traduisantes ${ }^{7}$. Pourtant, ce qui soutient le discours de «l'autorisation limitée» c'est une conception implicite de la textualité (et en même temps de «l'auteur»). Cette définition se situe entre deux pôles: d'une part le commentaire (le maintien des textes fondateurs) et d'autre part la rhétorique (la production d'un nouveau texte à partir de l'ancien). On peut mieux comprendre le discours de l'autorisation en situant ses termes dans l'un ou l'autre de ces deux paradigmes.

\section{LA SIGNATURE DU TRADUCTEUR ; LES PRATIQUES DE L'ATTRIBUTION}

Qu'en est-il du nom du traducteur? Peut-on voir dans l'histoire des pratiques de l'attribution à partir de la Renaissance une indication de la signification qui est accordée au travail du traducteur?

La réponse à cette question, en apparence simple, est assez complexe. Les pratiques d'attribution pour les auteurs autant que pour les traducteurs ne se sont pas fixées de façon définitive avant la fin du $17^{\mathrm{e}}$ siècle. S'il est vrai que «depuis la Renaissance un livre ne porte à conséquence que parce qu'il est référé à un sujet qui l'a commis» (Compagnon: 327 ) le caractère obligatoire du nom de l'auteur sur la page titre ainsi que les autres protocoles de la «périgraphie» du livre sont assez largement négligées jusqu'alors. Un grand nombre de livres portent des initiales ou des formules conventionnelles (la profession ou les titres de noblesse de l'auteur) à la place du nom de l'auteur ou du traducteur. En même temps, des œuvres présentées sous le nom d'un «auteur» sont souvent des traductions non avouées (Chas. Whibley : 24).

Tout indique en fait qu'à la Renaissance c'est moins la présence ou l'absence du nom du traducteur qui est significative que l'identité du dédicataire. Toute la matière liminaire du livre, en fait (dédicace, préface, vers honorifique, emblème et slogan), dit l'identité du livre et lui sert de protection.

L'importance des dédicataires indiquait le prestige relatif des écrits. En France, le nom du Roi servait à protéger plus souvent qu'autrement des textes anciens, tandis que le nom des femmes de la famille royale protégeait les æuvres traduites des langues vulgaires (Guillerm: 28). En Angleterre, le réseau des dédicataires est essentiel à la production de traductions, mais la distinction entre les langues classiques et des langues vernaculaires n'est pas pertinente puisqu'une bonne partie des versions anglaises sont produites à partir de versions françaises intermédiaires (Rosenberg: 155).

Ce n'est qu'après la Renaissance donc que le nom du traducteur devient un indice significatif. Qu'est-ce qu'il signifie, au fait? Plutôt que le degré de subjectivité investie par le traducteur, la présence du nom du traducteur en vient à signaler la valorisation de certains types de discours. C'est donc d'après le critère de genre que se démarquent les pratiques de l'attribution. Il semble assez clair, par exemple, que ce sont les ouvres en prose et en particulier le roman - surtout à partir du $18^{\mathrm{e}}$ siècle — pour lesquelles le nom 
du traducteur est systématiquement négligé8. En général c'est la qualité de la publication qui déterminera le soin apporté à fournir les indices de la source du texte. Plus l'ouvrage est bon marché, moins il y aura indication de l'origine du texte. C'est toujours le cas aujourd'hui.

Le nom du traducteur fait partie du droit moral des traducteurs - et n'a que dans quelques pays constitué une obligation légale pour les éditeurs. C'est parce que le nom du traducteur n'est pas nécessaire à la circulation et à la classification des livres qu'il a tendance à se faire oublier. Si le nom du traducteur figure sur une première édition d'un livre, il ne figurera pas toujours dans les éditions successives (surtout si l'auteur appartient au domaine public). Qui plus est, la simple mention du nom du traducteur peut prendre des dimensions très diverses selon la grandeur physique de la signature du traducteur et son emplacement.

Il arrive parfois que le nom du traducteur remplace celui de l'auteur original dans la présentation du livre. Cette pratique, relativement rare, signale l'appropriation de l'œuvre par la culture d'accueil'. Il s'agit d'une pratique parallèle à celle proposée par les théoriciens des «Belles infidèles» et l'expression d'une traduction commandée par «la rhétorique». Le traducteur devient le représentant autorisé de l'auteur; l'auteur (ancien, lointain) parle à travers la voix de celui qui sait actualiser son œuvre pour un public contemporain.

Présence incertaine et fuyante, le nom du traducteur est moins l'expression d'une subjectivité dans le texte que l'indice de la hiérarchisation des discours dans un contexte donné. Si le nom du traducteur figure aujourd'hui très généralement dans les livres imprimés, il ne paraît que rarement dans les autres formes de publications.

\section{LA SIGNIFICATION JURIDIQUE DU NOM DU TRADUCTEUR; LA PROPRIÉTÉ INTELLECTUELLE}

Le nom du traducteur est le lieu d'une responsabilité contractuelle avec l'éditeur et, en fin de compte, d'une responsabilité juridique. Le lien du traducteur à son texte est défini par les lois sur la propriété intellectuelle et le droit d'auteur.

Le discours sur la traduction entretient depuis toujours un rapport hautement ambigu, voire névrotique, à la Loi. Il y a en effet confusion constante entre la dimension morale de l'activité traduisante et sa dimension proprement juridique. Les traducteurs de la Renaissance et de l'époque classique ont entretenu cette confusion en faisant référence à des «lois» qu'ils interprétaient à leur guise ${ }^{10}$.

La traduction est cependant liée à la loi de façon effective. Jugés coupables de transgressions des dogmes religieux, des traducteurs (Tyndale, Etienne Dolet) ont été punis par la mort. La traduction littérale de la Bible ainsi que la pratique de la traduction collective ont été dans la tradition chrétienne des stratégies destinées à éviter la responsabilité du texte traduit (Schwarz 1944). La production de traductions est une obligation décrétée par la loi dans les pays à administration bilingue, comme le Canada; cependant, les lois (curieusement pudiques sur ce plan) ne font pas mention explicite de la traduction ni des normes auxquelles elle doit se conformer ${ }^{11}$.

Les lois sur la propriété intellectuelle réglementent la pratique moderne de la traduction. C'est parce que le texte est la propriété d'un auteur que le traducteur est tenu de respecter ce texte. De façon surprenante, cependant, la perspective des lois sur le droit d'auteur vise plus à protéger «l'œuvre» du traducteur qu'à la contraindre à respecter des normes pré-établies.

En effet, les paramètres qui définissent la légitimité de la traduction d'après les lois du droit d'auteur sont radicalement différentes de celles que ressasse la théorie de la traduction depuis toujours. Les lois françaises et anglaises, bien que différentes de plusieurs 
points de vue dans leur conception du droit d'auteur, ont toujours reconnu l'existence du texte traduit comme une cuvre autonome produite par son propre «auteur». «Il a toujours été admis que le traducteur fait preuve d'originalité dans le choix des expressions pour rendre au mieux en une langue le sens du texte en une autre langue» (Colombet: 46). «Le traducteur est donc considéré comme un auteur pour sa propre traduction» (Monnet: 370). Les lois n'ont pas toujours reconnu le traducteur de façon explicite, cependant. Dans la loi britannique, c'est la jurisprudence qui définit les droits du traducteur. Les lois françaises et américaines ainsi que les lois internationales font explicitement mention du traducteur ${ }^{12}$. La jurisprudence française est de loin celle qui est la plus loquace sur les questions de traduction.

La conception moderne du droit d'auteur repose essentiellement sur les notions de propriété élaborées par John Locke : le droit à la propriété de l'œuvre est un droit naturel au fruit de son travail. La loi n'a donc jamais protégé l'originalité mais bien le résultat d'un effort (Ploman: 25-26). D'un droit à la reproduction de l'œuvre (le droit économique), le droit d'auteur est devenu également un droit moral (un droit de «personnalité», disent les Français (Colombet : 15)).

Même si le traducteur est auteur de son œuvre, il l'est toujours «sans préjudice aux droits de l'auteur». Cela veut dire que la question de l'adéquation entre les deux textes est définie uniquement en termes du respect du droit moral de l'auteur. L'œuvre du traducteur est donc à la fois le licu de son propre droit et celui (antérieur) de l'auteur. Cette double obligation peut évidemment poser certains problèmes et d'abord celui, théorique, du fondement même de la notion du droit d'auteur. Nous savons que les lois sur le droit d'auteur ne protègent que la formulation des idées et non pas les idées elles-mêmes. «Les idées font partie du domaine public, et comme telles sont susceptibles d'une libre exploitation. C'est l'originalité de la forme qui fera de l'œuvre la propriété de son auteur» (Dock : 18) ${ }^{13}$. Si, comme il est bien reconnu, la traduction implique un changement formel obligatoire, quelle partie de l'cuvre originale est protégée dans la traduction? William Braithwaite, analyste juridique, a posé la question dans ces termes:

Yet many derivative works by their very nature take on a different shape or form from that of the underlying work. Therefore if a copyright owner is given the exclusive right to produce derivative works based on his underlying work, something other than simply the form or expression of his creative effort is being protected (Braithwaite : 204).

Derrida exprime le problème ainsi :

Si une première conséquence est bonne ( $\mathrm{du}$ fait que le droit $\mathrm{d}$ 'auteur ne protège que la forme) puisque c'est cette forme qui définit l'originalité de la traduction, une autre conséquence en pourrait être ruineuse car elle devrait conduire à abandonner ce qui distingue l'original de la traduction si, à l'exclusion de l'expression, il revient à une distinction de fond. À moins que la valeur de composition, si peu rigoureuse qu'elle soit, ne reste l'indice du fait qu'entre l'original et la traduction le rapport n'est ni d'expression ni de contenu mais d'autre chose au-delà de ces oppositions (Derrida : 241).

Les lois n'ont jamais formulé de façon explicite le type d'équivalence qui doit exister entre la traduction et son original. Les quelques tentatives dans ce sens (surtout dans la jurisprudence britannique) se sont avérées inutiles et les juges ont dû par la suite avoir recours aux «experts» qui eux étaient chargés d'établir la qualité d'une traduction. En fait, la loi se contente d'établir de façon parallèle mais hiérarchisée les droits de l'auteur et du traducteur ${ }^{14}$. La plupart des causes impliquant des traductions dans les traditions britanniques, américaines, françaises et canadiennes concernent des droits économiques 
et surtout la vente de traductions non autorisées. Mais ces paradoxes de la loi sur le droit d'auteur ressortent clairement d'un conflit particulièrement intéressant qui a opposé les éditions Gallimard aux héritiers d'Alexandre Vialatte ${ }^{15}$.

Pour la publication de l'œuvre complète de Kafka dans les prestigieuses éditions de la Pléiade, les éditions Gallimard ont signé un contrat avec le traducteur bien connu Alexandre Vialatte, s'engageant à utiliser ses traductions déjà publiées. Notant «de nombreuses inexactitudes, maladresses, faux sens, contresens, obscurités, et même des omissions dans les traductions», Claude David, engagé par Gallimard pour préparer l'édition, suggéra que les textes soient révisés. Pierre Vialatte, héritier de son père, refusa de faire modifier les traductions au nom de l'intégrité de l'œuvre de son père. En l'absence d'une «voix» représentant l'œuvre de Kafka, les éditions Gallimard ont été obligées de respecter leur contrat avec Vialatte - malgré la preuve des «fautes» de traduction et malgré un jugement de 1961 qui déclare que «Des omissions ou des additions dans une traduction constituent une atteinte au droit moral de l'auteur, au respect de son œuvre» (art. 6 de la loi đu 11 mars 1957). C'est que le texte lui-même n'a pas de droits. La loi protège non pas une quelconque correspondance entre deux textes mais bien la priorité de certaines «voix» qui parlent pour le texte. (Les éditions Gallimard ont cependant reçu la permission de remédier à quelques omissions, mais seulement dans l'appareil critique du texte.)

Le paradoxe ultime de la protection légale des traducteurs c'est que plus souvent qu'autrement le statut accordé par la loi n'est nullement reflété dans les pratiques des éditeurs ${ }^{16}$. Dans une intervention ironique, Élizabeth Janvier suggère que les notions «d'auteur» et «d'œuvre» associées à la traduction ne rendent nullement service au traducteur. Au contraire, les éditeurs profitent de la conception de la vocation littéraire des traducteurs pour mieux les abuser (Janvier 1981). Dans ce sens on peut se demander si l'inclusion espérée de la mention des traducteurs dans la nouvelle loi sur les droits d'auteurs au Canada va effectivement améliorer le sort des traducteurs. Il faudrait qu'un litige bien publicisé attire l'attention des éditeurs sur les droits réels (et non seulement potentiels) des traducteurs (droits moraux autant que droits économiques).

Les efforts des associations professionnelles des traducteurs (littéraires, en l'occurrence) pour obtenir un éclaircissement de leurs droits face aux éditeurs, aux tribunaux et aux instances étatiques font partie d'un large mouvement de revendication de la part des associations des créateurs au Canada et ailleurs. Les demandes de ces organismes concernent non seulement le statut fiscal du créateur mais aussi son droit moral. Ce mouvement aboutira peut-être à un nouveau partage de pouvoirs entre éditeurs, créateurs et État, semblable dans ses dimensions à celui effectué à la fin du $18^{\mathrm{e}}$ siècle et qui a vu disparaître les privilèges des libraires.

Le poids du traducteur est actuellement relativement indéterminé, et défini par les rapports de force en place. Ainsi, la «part» du traducteur (dans les contrats de traduction, mais aussi dans les programmes de rétribution du prêt public ou de reprographie, par exemple) est une quantité négociable. Cette indétermination signale peut-être une mutation en cours...

\section{LA THÉORIE, AUJOURD'HUI}

Les théories modernes de la traduction (celles qui sont ancrées dans des champs de savoir disciplinaire - par opposition aux discours traditionnels) font état également de changements importants dans le sens accordé au nom du traducteur comme sujet de l'écriture. Il faudrait consacrer à cette évolution une étude substantielle, mais esquissons ici quelques repères. 
La littérature comparée est la première discipline moderne à avoir adopté les traductions comme objet d'étude. Les importants travaux consacrés à la traduction (au début du siècle) définissent le sujet de la traduction comme un sujet collectif (national) et du fait même comme un sujet de méconnaissance.

Tout traducteur subit, qu'il le veuille ou non, l'influence secrète du goût propre à sa nation ; malgré lui, il fait passer dans sa version ses propres habitudes intellectuelles, ses façons de sentir : ainsi l'image qu'il donne d'un auteur étranger ne saurait être qu'inexacte ${ }^{17}$.

Bien que liée à l'étude d'une période précise (le romantisme), cette conception soutient une grande partie des études littéraires consacrées à la traduction. Les études littéraires s'intéressent d'ailleurs à la traduction aux moments justement où la cote de l'auteur est en baisse et où on met en question la souveraineté du «grand» écrivain, en interrogeant davantage les «voix mineures» et l'infrastructure institutionnelle de la littérature.

$\mathrm{Au}$ début des années soixante, la linguistique structurale a donné un grand élan aux études du processus de la traduction mais elle a en même temps rigoureusement exclu de ce processus tout soupçon de subjectivité. Le sujet de la traduction a été éliminé; le traducteur et son contexte social n'avaient aucune pertinence pour ce modèle linguistique. Avec la sociolinguistique, cependant, et la diversification croissante des perspectives d'étude de la traduction (l'herméneutique, la psychanalyse, les théories du texte, etc.) la question de la subjectivité est devenue de nouveau problématique. Le grand intérêt du travail à la fois des théoriciens du polysystème (Hermans 1985) et d'Antoine Berman (1984) est de reconnaître à la traduction une composante idéologique spécifique (sans aller jusqu'à identifier «auteur» et «traducteur» comme le font certaines théories textuelles). Pour le premier groupe, le sujet de la traduction est jusqu'à un certain point un sujet passif, contraint par les modèles d'écriture qui lui sont disponibles. Pour Berman, le traducteur est davantage le créateur d'un savoir propre, agissant parfois contre les modèles dominants (Berman : 286-287).

L'avantage de la perspective de Berman c'est d'une part de rendre compte du pouvoir d'intervention des traductions dans le monde littéraire (le pouvoir d'innovation) et d'autre part de faire de la traduction l'expression d'un projet (et non plus d'une simple tâche). En même temps les nombreux travaux empiriques qui sont inspirés du modèle du polysystème contribuent à une meilleure compréhension de la «part» effective du traducteur, en soulignant la complexité des rapports d'interaction entre les divers systèmes en place.

\section{Conclusion}

Scruté à partir de plusieurs postes d'observation distincts, le nom du traducteur se révèle porteur de significations diverses et parfois paradoxales. Il nomme un sujet historiquement constitué, une position dont le contenu précis sera variable selon les époques et les régimes textuels; il indique l'insertion du texte dans une hiérarchie de pouvoirs (celui de l'auteur, celui du genre); il désigne pour le discours critique traditionnel (celui de l'autorisation limitée) soit le degré zéro de la fỉdélité (dans une culture du commentaire) soit le supplément du style (dans une culture de la rhétorique); il est le lieu d'un pouvoir et d'une responsabilité juridiques, définis de façon symétrique à ceux détenus par l'auteur, mais peu respectés dans la pratique; et enfin il désigne pour la théorie moderne (disciplinaire) des conceptions parfois contradictoires de la subjectivité dans l'écriture.

De ces constatations d'ordre très divers, on peut conclure que la signification du nom du traducteur relève nécessairement des enjeux les plus larges concernant l'écriture dans un contexte donné. C'est en ouvrant les études de l'histoire et la théorie de la traduction au plus grand nombre de niveaux d'analyse (sociaux, économiques, idéologiques, 
textuels) que nous pourrons mieux comprendre à la fois le sujet traducteur et l'environnement complexe qui le façonne.

Notes

* Cette recherche a été entreprise à l'aide d'une subvention du Conseil de la recherche en sciences humaines du Canada.

** Auteur et traducteur réfèrent ici à des positions et non pas à des individus. Ainsi renonçons-nous à utiliser les formes féminisées.

1. Michel Foucault (1969) : «Qu'est-ce qu'un auteur?», Bulletin de la Société française de philosophie, 63 , n 3. Repris dans Language, Counter-Memory, Practice, trans. Don Bouchard \& S. Simon, Cornell University Press, 1977.

2. Cette esquisse historique s'inspire très largement de l'étude très importante de Gianfranco Folena (1973) : «Volgarizzare e tradurre», La traduzione, saggi et studi, Triest, Linze, pp. 57-120.

3. Pour une étude de la transition entre la conception scholastique de l'auctoritas et la conception moderne de l'auteur, voir Minnis (1984) : Medieval Theory of Authorship, London, Scholar Press.

4. Auteurs et traducteurs vendent de la même manière leur manuscrit aux éditeurs, bien que certains éditeurs se spécialisent dans les traductions. Auteurs et traducteurs peuvent exceptionnellement acquérir des «privilèges» qui leur donnent le monopole sur un sujet en particulier.

Alexander Nevill even obtained the prohibition of every translation of Livy except his own... This was standard practice throughout Europe at this time. According to G.H. Putnam, the possessor of a «privilege" for a book in a particular subject was regarded as holding a monopoly of the subject. In the case of an edition of a classical author, the privilege thus barred the production of any other edition. (Sheavyn : 77)

5. La figuration métaphorique de la traduction est d'une stabilité étonnante. La figure de Dryden reprend celle-ci, énoncée 150 ans plus tôt

Car tout ainsi, comme ung deffricheur qui ... rend par son extreme travail et continuelle sueur labourables et fertiles les lieux ... n'en reçoit pourtant aucun autre proffit ni advantaige, que le salaire commun de ses journées: mais en retoument et sont delivrez au seigneur proprietaire le fruitz et revenu : tout ainsi n'est attribuée grande louenge a ung translateur d'histoire ou autre escriture, quelque diligence qu'il y ait mise : ... et que combattre en camp cloz, dont l'on n'ose oultrepasser les barrieres, ne soit moins louable, que d'estre en pleine campaigne ou l'on peult s'eslargir et courrir à bride abbatue.

Antoine Macault (1541) : (cité Norton : 231)

Pour des études de la figuration métaphorique de la traduction, voir Theo Hermans (1985): «Images of Translation: Metaphor and Imagery in the Renaissance Discours on Translation» in Hermans, Ed., The Manipulation of Literature, Croom Helm; Serge Gavronsky (1977): «The Translator: From Piety to Cannibalism», Sub-stance 16, pp. 53-62.

6. En définissant la légitimité de la traduction d'après des critères historiques, de Blignières rejoint les analyses de E. Egger (très citées au $19^{\mathrm{e}}$ et au début du $20^{\mathrm{e}}$ siècle) sur la nature essentiellement historique du traduisible. Egger suggère que le moment de la traduisibilité maximale d'Homère aurait été le Moyen Âge français. E. Egger (1869) : l'Hellénisme en France, t. 1, Paris, pp. 189 ss.

7. Ce commentaire de Defauconpret est une exception tout à fait éloquente :

Je viens d'apprendre que Lady Morgan désavoue la traduction que j'ai fait paraître de son dernier roman intitulé Florence Macarthy.

Lady Morgan, bien pénétrée de tout son mérite, croit que ses ouvrages doivent être traduits avec le même respect religieux que ceux d'Horace ou de Tacite? J'ai le malheur de ne pas être tout à fait du même avis. Je crois qu'en faisant passer un roman d'une langue dans une autre, le premier devoir d'un traducteur est de le mettre en état de plaire aux nouveaux lecteurs qu'il veut lui procurer. (Mots mis en italiques par l'auteur.)

Lettre publiée dans le Journal des débats, 1819. Citée dans Horguelin, Anthologie de la manière de traduire, p. 154.

8. C'est la conclusion de l'étude de Lambert, d'Hulsdt, et van Bragt pour la période 1800-1850 en France : «The translation of a play or a poem was subject to rules which the translation to a novel or short story could ignore with impunity". "Translated literature in France, 1800-1850" in The Manipulation of Literature, Studies in Literary Translation, Ed. Theor Hermans, Croom Helm, London \& Sydney (p. 158). Parmi ces règles se trouvera l'obligation de noter le nom du traducteur ainsi que la source précise de l'original et ses traductions antérieures. Cette conclusion est corroborée notamment par les listes d'attributions foumies dans The Cambridge Bibliography of English Literature où on remarque une diminution importante des attributions pour les romans traduits après 1700 . On ne trouve pas cette même diminution, par contre, dans le Manuel bibliographique de Lanson qui donne par ailleurs une liste impressionnante de traductions 
tirées de tous les genres. Peut-on attribuer cette différence aux méthodes bibliographiques ou à l'inclusion de la part de Lanson uniquement de romans considérés importants?

9. Dans le contexte québécois, signalons le cas des traductions thêâtrales (étudiées par Annie Brisset) qui sont signées en page couverture uniquement du nom de leurs traducteurs/adaptateurs (Michel Tremblay, JeanClaude Germain, etc.) Le seul cas semblable du côté anglo-canadien serait la traduction des Anciens Canadiens de Philippe Aubert de Gaspé dont seul le nom du traducteur, Charles G.D. Roberts, apparaît sur la couverture du New Canadian Library Edition. De manière générale, le nom du traducteur est davantage mis en importance (par sa présence sur la page couverture) du côté anglo-canadien que du côté québécois.

10. Pour Norton, les «règles» de la traduction sont «a great wistful lie of the Renaissance», p. 14. La formulation du Digeste justinien contre le littéralisme a cependant été appliquée au moins une fois : il s'agit du cas, présenté par Norton, des lecteurs royaux : «magis sensus quam verba spectatur»; «melius est sensum magis quam verba amplectis.

11. Ni la loi canadienne sur le statut des langues officielles ni la loi 101 du Québec ne mentionne la traduction de façon explicite. L'existence d'une assurance-responsabilité pour traducteurs implique bien sûr les possibilités pour un traducteur d'être l'objet de poursuites judiciaires. La STQ a offert cette assurance durant un certain temps mais l'a abandonnée faute d'intérêt. Puisque les traductions produites au cours de l'emploi sont considérées la propriété de l'employeur, c'est l'employeur qui en général s'assure.

12. Les traducteurs ne sont mentionnés explicitement que dans quelques lois nationales et internationales sur le droit d'auteur. (France: loi du 11 mars 1957, article 4; USA : US Copyright Act of 1976, Sec. 101 : definition of «derivative works». En Angleterre comme au Canada - jusqu'à la refonte de la loi, maintenant en cours - la protection est implicite et assurée par la jurisprudence: pour l'Angleterre Byrne v. Statist. Comp. England, 1914 ; pour le Canada Pasickniak vs Dojacek, Manitoba, 1928; la Convention de Berne, article 2, appendice 2: protection de traductions.) Les traducteurs sont également touchés par les dispositions des lois sur les droits d'auteur qui concernent le travail effectué à l'emploi d'un autre. Dans les lois actuelles, le travail effectué pour un employeur appartient de droit à l'employeur. D'après Mounin, la révision de Berlin de la convention de Berne (1908) constitue la norme fondamentale, morale et juridique du traducteur comme auteur. La convention de Genève (1952) maintient cette protection mais élimine la mention explicite du traducteur comme auteur. Il est difficile, croit Witthaus, de croire que cette omission a été involontaire. Elle refléterait indirectement la volonté et l'insistance d'auteurs d'œuvres originales de se réserver le titre exclusif d'auteurs (Witthaus : 231)

13. C'est encore l'injonction horatienne qui sert ici de référence historique. Marie-Claude Dock suggère que l'on retrouve dans la délimitation entre le domaine public et le domaine privé l'énonciation dans l'Antiquité d'une forme embryonnaire de la conception moderne du droit d'auteur. D'après Horace, cependant, la traduction «fidèle» serait automatiquement exclue du domaine du privé puisque on ne peut pas ainsi accéder à l'originalité. «Vous ferez d'une matière prise au domaine public votre propriété privée si vous ne vous attardez pas à faire le tour de la piste banale et ouverte à tous, si vous ne vous appliquez pas à rendre, traducteur fidèle, le mot par le mot...» (cité dans Compagnon : 153).

14. Une note suivant le jugement du Trib. de Gr. Inst. de Lyon, 16 fév. 1961, Gaz. Pal. mentionne cette difficulté de façon explicite : «Le droit moral de l'auteur au respect de son ouvre ... s'applique évidemment aux traductions, qui doivent être fidèles et ne peuvent comporter des coupures sans l'autorisation de l'auteur. La détermination des limites au-delà desquelles les adaptations nécessitées par toute traduction dénaturent l'œuvre est plus délicate.»

15. Trib. de gr. inst. de Paris, 25 sept. 1974. Revue internationale du droit d'auteur, LXXIX, 1975.

16. «On peut se demander si l'assimilation juridique aux œuvres originales des adaptations, notamment des traductions, trouve un écho dans la pratique ou si une certaine dépréciation de la prestation de l'adaptateur (par exemple, le traducteur) par rapport à la prestation de l'auteur original, n'a pas pour effet d'entraîner un affaiblissement et un respect moindre des droits de l'adaptateur dans la pratique. À cet égard, il convient de relever les doléances souvent justifiées à plus d'un titre des traducteurs qui - indépendamment des questions de rémunération - se sentent fréquemment lésés dans le domaine du droit moral, notamment en ce qui concerne la mention du nom.» Adolf Dietz (1976) : le Droit d'auteur dans la communauté européenne, Commission des communautés européennes, Bruxelles, p. 46.

17. Paul Hazard (1921): «L'invasion des littératures du Nord dans l'Italie du XVIIIe siècle», la Revue de littérature comparée, I, p. 46. 


\section{BIBLIOGRAPHIE}

AMOS, Flora Ross (1979) : Early Theories of Translation, New York, Farrar Straus and Giroux (rpt. of 1920 edition).

BENNETT, H.S. (1952) : English Books and their Readers, vol. I, II, III, Cambridge.

DE BLIGNIERES, Auguste (1851) : Essai sur Amyot et les traducteurs français au XVIe siècle, Paris.

DU BELLAY, Joachim (1878) : Deffence et illustration de la langue françoyse, éd. E. Person, Paris.

BERMAN, Antoine (1984) : l'Épreuve de l'étranger, Gallimard.

CAVE, Terence (1979): The Cornucopian Text: Problems of Writing in the French Renaissance, Oxford, Clarendon Press.

CHARLES, Michel (1985): l'Arbre et la source, Paris, Seuil.

COMPAGNON, Antoine (1979) : la Seconde main, Paris, Seuil.

DERRIDA, Jacques (1984): «Languages and Institutions of Philosophy», Recherches sémiotiques / Semiotic Inquiry, vol. 4, pp. 91-154.

DERRIDA, Jacques (1985): «Des tours de Babel» in Difference in Transiation, Ed. Joseph Graham, Comell University Press.

DRAPER, J.W. (1921) : *The Theory of Translation in the 18th Century», Neophilologus 6, pp. 241-254.

EGGER, Émile (1869) : l'Hellénisme en France, t. 1, 2, Paris (rept. Burt Franklin, NY).

FOLENA, Gianfranco (1973) : «Volgarizzare e tradurre», La traduzione, saggi e studi, Trieste, Linze, pp. 57120.

FORSTER, Leonard (1970): The Poet's Tongues. Multilingualism in Literature, Cambridge University Press.

GENETTE, Gérard (1982): Palimpsestes, Paris, Seuil.

GUILLERM, Luce (1980) : «L'auteur, les modèles, et le pouvoir; ou la topique de la traduction au XVI siècle», Revue des Sciences humaines, 52.

HERMANS, Theo, Ed. (1985): The Manipulation of Literature, Croom Helm.

HORGUELIN, Paul (1981) : Anthologie de la manière de traduire, Montréal, Linguatech.

JONES, R.F. (1953): The Triumph of the English Language, Stanford University Press.

KELLY, L.G. (1979) : The True Interpreter, Oxford, Basil Blackwell.

LANSON, Gustave (1920): Manuel bibliographique de la littérature française moderne, $2^{\mathrm{e}}$ éd., Paris, Hachette.

MINNIS, A.J. (1984): Medieval Theory of Authorship, London, Scholar Press.

MONFRIN, J. (1964) : «Humanisme et traductions au Moyen Âge», in l'Humanisme médiéval dans les littératures romanes $d u 12^{e}$ au $14^{e} s$., Paris, Klincksieck.

MOSER, Walter (1985) : «Les pulsations de la traduction», META, 31:1 (mars), pp. 7-18.

NORTON, Glyn P. (1984): The Ideology and Language of Translation in Renaissance France, Genève, Droz.

PERROT D'ABLANCOURT, Nicolas (1972): Lettres et préfaces critiques, éd. Roger Zuber, Paris, Didier.

PÉPIN, Jean : l'Herméneutique ancienne», Poétique 23, pp. 291-300.

ROSENBER, Eleanor (1955) : Leicester, Patron of Letters, New York, Columbia University Press.

SHEAVYN, Phoebe, Rev. J.W. SAUNDERS (1967): The Literary Profession in the Elizabethan Age, Manchester University Press.

SCHWARZ, W. (1944): «The Meaning of Fidus Interpres in Medieval Translation», The Journal of Theological Studies, vol. 45, pp. 368-373.

STEINER, T.R. (1975) : English Translation Theory 1650-1800, Van Gorcum, Assen/Amsterdam.

TERRACINI, B. (1957) : «Il problema della traduzione», in Conflitti di lingua et di cultura, Venezia.

WHIBLEY, Chas. (1964): "Translators» in The Cambridge History of English Literature, vol. IV, Ed. A.W. Ward, A.R. Waller, Cambridge University Press.

WELLS, Margaret B. (1980): "What Did Du Bellay Understand by Translation ?», Forum for Modern Language Studies, 16, pp. 175-185.

WEINSHEIMER, Joel (1984): Imitation, Routledge and Kegan Paul.

YOUNG, Edward (1759): Conjectures on Original Composition, London, rpt. The Scholar Press Limited, 1966.

ZUBER, Roger (1968): «Les «Belles Infidèles» et la formation du goût classique, Paris, Armand Colin.

Traduction et droits d'auteur

BIRRELL, Augustine (1899): Seven Lectures on the Law and History of Copyright in Books, London (Rothman Reprints, New York, 1971).

BRAITHWAITE, William J. (1982): «Derivative Works in Canadian Copyright Law», Osgoode Hall Law Journal, vol. $20, \mathrm{n}^{\circ} 2$, June, pp. 192-231.

CAMMAERT, G. (1985): «Le traducteur et le droit d'auteur» (en Belgique), META, 30:4, décembre, pp. 400. 402.

COLOMBET, Claude (1976): Propriété littéraire et artistique, Dalloz.

DOCK, Marie-Claude (1963) : Étude sur le droit d'auteur, Paris. 
KAFKA, Dossier (1983) : «Les nouvelles traductions du procès», les Nouvelles littéraires.

MONNET, Pierre (1962) : Dictionnaire pratique de propriété littéraire, Paris, Cercle de la librairie.

PLOMAN, Edward W. and L.C. HAMILTON (1980) : Copyright, Routledge \& Kegan Paul

PUTNAM, George Haven (1896): Books and their Makers in the Middle Ages. A history of the concept of literary property. New York, Hilary House Publishers (rpt. 1962).

WITTHAUS, Rodolfo (1981): Regimen Legal de la Traduccion y del Traductor Publico, Buenos Aires, Abeledo Perrot.

«Recommendation on the legal protection of translators and translations and the practical means to improve the status of translators», UNESCO, adopted by the General Conference at its Nineteenth Session, Nairobi, 22 Nov. 1976.

De Gutenberg à Télidon, Livre blanc sur le droit d'auteur, ministère des Communications, Consommation et Corporations Canada, 1984

«For a New Copyright Law», Conference of Quebec Arts Creators' Associations. Brief presented to Hon. André Ouellet et Hon. Francis Fox, November 1982.

Pasickniak v. Dojacek. Court of Appeal, Manitoba (1928) 2 D.L.R. 545.

Byme v. Statist. Company, Court of Appeal, England (1914) K.B.D. 622. 